\title{
An improved formulation of the relativistic hydrodynamics equations in 2D Cartesian coordinates
}

\author{
Thorsten Kellerman $^{1}$, Luca Baiotti ${ }^{1,2}$, Bruno Giacomazzo ${ }^{1}$ \\ and Luciano Rezzolla ${ }^{1,3,4}$ \\ ${ }^{1}$ Max-Planck-Institut für Gravitationsphysik, Albert Einstein Institut, Golm, Germany \\ ${ }^{2}$ Graduate School of Arts and Sciences, University of Tokyo, Komaba, Meguro-ku, \\ Tokyo, 153-8902, Japan \\ ${ }^{3}$ Department of Physics, Louisiana State University, Baton Rouge, LA 70803, USA \\ ${ }^{4}$ INFN, Sezione di Trieste, Trieste, Italy
}

Received 22 June 2008, in final form 18 August 2008

Published 31 October 2008

Online at stacks.iop.org/CQG/25/225007

\begin{abstract}
A number of astrophysical scenarios possess and preserve an overall cylindrical symmetry when also undergoing a catastrophic and nonlinear evolution. Exploiting such a symmetry, these processes can be studied through numericalrelativity simulations at smaller computational costs and at considerably larger spatial resolutions. We present here a new flux-conservative formulation of the relativistic hydrodynamics equations in cylindrical coordinates. By rearranging those terms in the equations which are the sources of the largest numerical errors, the new formulation yields a global truncation error, which is one or more orders of magnitude smaller than those of alternative and commonly used formulations. We illustrate this through a series of numerical tests involving the evolution of oscillating spherical and rotating stars, as well as shock-tube tests.
\end{abstract}

PACS numbers: 04.25.D, 04.40.Dg

\section{Introduction}

Numerical simulations assuming and enforcing axisymmetry are particularly useful to study, at higher resolution and smaller computational costs, those astrophysical scenarios whose evolution is expected to possess and preserve such a symmetry. On the other hand, the numerical solution of systems of equations expressed in coordinates adapted to the symmetry has often posed serious difficulties, because of the coordinate singularity present on the symmetry axis. The 'cartoon' method, proposed by Alcubierre et al [1], allows us to exploit the advantages of reduced computational resource requirements, while adopting Cartesian coordinates, which are non-singular. 
The cartoon method proves particularly useful in the numerical evolution of smooth functions, such as the metric quantities of the Einstein equations. However, because of the interpolations necessary to impose the axisymmetric conditions on a Cartesian grid, the cartoon approach is not considered to be accurate enough to describe the shocks which generically develop when matter is present. As a consequence, general-relativistic codes employing the cartoon method have adopted cylindrical coordinates for the evolution of the matter (and magnetic field) variables [2-8]. All the cited works adopt the same formulation for the hydrodynamical equations in cylindrical coordinates. In the present paper, we propose a slightly different formulation, which has proven to reduce the numerical errors, especially in the vicinity of the symmetry axis.

More specifically, we have written the Whisky2D code, which solves the generalrelativistic hydrodynamics equations in a flux-conservative form and in cylindrical coordinates. This, of course, brings in $1 / r$ singular terms, which must be dealt with appropriately. In the above-referenced works, the flux operator is expanded, and the $1 / r$ terms, not containing derivatives, are moved to the right-hand side of the equation (the source term), so that the lefthand side assumes a form identical to that of the three-dimensional (3D) Cartesian formulation. We call this the standard formulation. Another possibility is not to split the flux operator and to redefine the conserved variables, via a multiplication by $r$. We call this the new formulation. The new equations are solved with the same methods as in the Cartesian case. From a mathematical point of view, one would not expect differences between the two ways of writing the differential operator, but, of course, a difference is present at the numerical level. Our tests show that the new formulation yields results with a global truncation error, which is one or more orders of magnitude smaller than those of alternative and commonly used formulations.

Here, we perform a series of tests to ascertain the convergence behaviour of the two formulations. We then show that the new formulation produces results which are generally more accurate, with a truncation error, which can be several orders of magnitude smaller.

The paper is organized as follows. In section 2, we recall the essentials of the cartoon approach for the evolution of the geometrical variables, while in section 3, we review the flux-conservative formulation of relativistic hydrodynamics. We write down the relativistic flux-conservative hydrodynamics equations for axisymmetric formulations, and illustrate the two possible ways to write the singular term. In section 4, we present several tests that compare the two formulations. We begin with the conservation of rest mass and angular momentum in the Cowling approximation and in full-spacetime evolution. Then, the eigenfrequencies of uniformly rotating neutron-star models are compared with the results of a perturbative code. The last test examines the differences between the two formulations with respect to an analytic solution of an extreme shock case, which mimics the reflection of a cold and very fast gas at the symmetry axis.

We have used a spacelike signature $(-,+,+,+)$, with Greek indices running from 0 to 3 , Latin indices from 1 to 3 and the standard convention for the summation over repeated indices. Unless explicitly stated, all the quantities are expressed in the system of dimensionless units in which $c=G=M_{\odot}=1$.

\section{Evolution of the Einstein equations}

The logical and algorithmic structures of the Whisky2D code presented here follow closely those of the CCATIE [9] and Whisky codes [10], which solve the same set of equations in 3D and using Cartesian coordinates. In what follows, we provide only a brief overview of the set of equations for the evolution of the fields (within the 'cartoon' prescription [1]) and for the 
evolution of the fluid variables, referring the interested reader to [9, 11, 12] for a more detailed discussion. As for the other codes mentioned above, Whisky2D is also based on the Cactus Computational Toolkit [13].

More specifically, we evolve a conformal-traceless ' $3+1$ ' formulation of the Einstein equations [14-16], in which the spacetime is decomposed into 3D spacelike slices, described by a metric $\gamma_{i j}$, its embedding in the full spacetime, specified by the extrinsic curvature $K_{i j}$, and the gauge functions $\alpha$ (lapse) and $\beta^{i}$ (shift), which specify a coordinate frame (see [17] for a general description of the ' $3+1$ ' split). The particular system which we evolve transforms the standard ADM variables as follows. The 3-metric $\gamma_{i j}$ is conformally transformed via

$$
\Phi=\frac{1}{12} \ln \operatorname{det} \gamma_{i j}, \quad \tilde{\gamma}_{i j}=\mathrm{e}^{-4 \Phi} \gamma_{i j},
$$

and the conformal factor, $\Phi$, evolved as an independent variable, whereas $\tilde{\gamma}_{i j}$ is subject to the constraint det $\tilde{\gamma}_{i j}=1$. The extrinsic curvature is subjected to the same conformal transformation, and its trace, $\operatorname{tr} K_{i j}$, is evolved as an independent variable. That is, in place of $K_{i j}$ we evolve

$$
K \equiv \operatorname{tr} K_{i j}=g^{i j} K_{i j}, \quad \tilde{A}_{i j}=\mathrm{e}^{-4 \Phi}\left(K_{i j}-\frac{1}{3} \gamma_{i j} K\right),
$$

with $\operatorname{tr} \tilde{A}_{i j}=0$. Finally, new evolution variables,

$$
\tilde{\Gamma}^{i}=\tilde{\gamma}^{j k} \tilde{\Gamma}_{j k}^{i},
$$

are introduced, defined in terms of the Christoffel symbols of the conformal 3-metric.

The Einstein equations specify a well-known set of evolution equations for the listed variables and are given by

$$
\begin{aligned}
& \left(\partial_{t}-\mathcal{L}_{\beta}\right) \tilde{\gamma}_{i j}=-2 \alpha \tilde{A}_{i j}, \\
& \left(\partial_{t}-\mathcal{L}_{\beta}\right) \Phi=-\frac{1}{6} \alpha K, \\
& \left(\partial_{t}-\mathcal{L}_{\beta}\right) \tilde{A}_{i j}=\mathrm{e}^{-4 \Phi}\left[-D_{i} D_{j} \alpha+\alpha\left(R_{i j}-8 \pi S_{i j}\right)\right]^{\mathrm{TF}}+\alpha\left(K \tilde{A}_{i j}-2 \tilde{A}_{i k} \tilde{A}_{j}^{k}\right), \\
& \left(\partial_{t}-\mathcal{L}_{\beta}\right) K=-D^{i} D_{i} \alpha+\alpha\left[\tilde{A}_{i j} \tilde{A}^{i j}+\frac{1}{3} K^{2}+4 \pi\left(\rho_{\mathrm{ADM}}+S\right)\right], \\
& \partial_{t} \tilde{\Gamma}^{i}=\tilde{\gamma}^{j k} \partial_{j} \partial_{k} \beta^{i}+\frac{1}{3} \tilde{\gamma}^{i j} \partial_{j} \partial_{k} \beta^{k}+\beta^{j} \partial_{j} \tilde{\Gamma}^{i}-\tilde{\Gamma}^{j} \partial_{j} \beta^{i}+\frac{2}{3} \tilde{\Gamma}^{i} \partial_{j} \beta^{j} \\
& \quad-2 \tilde{A}^{i j} \partial_{j} \alpha+2 \alpha\left(\tilde{\Gamma}^{i}{ }_{j k} \tilde{A}^{j k}+6 \tilde{A}^{i j} \partial_{j} \Phi-\frac{2}{3} \tilde{\gamma}^{i j} \partial_{j} K-8 \pi \tilde{\gamma}^{i j} S_{j}\right),
\end{aligned}
$$

where $R_{i j}$ is the three-dimensional Ricci tensor, $D_{i}$ is the covariant derivative associated with the 3-metric, $\gamma_{i j}$, 'TF' indicates the trace-free part of tensor objects and $\rho_{\mathrm{ADM}}, S_{j}$ and $S_{i j}$ are the matter source terms defined as

$$
\rho_{\mathrm{ADM}} \equiv n_{\alpha} n_{\beta} T^{\alpha \beta}, \quad S_{i} \equiv-\gamma_{i \alpha} n_{\beta} T^{\alpha \beta}, \quad S_{i j} \equiv \gamma_{i \alpha} \gamma_{j \beta} T^{\alpha \beta},
$$

where $n_{\alpha} \equiv(-\alpha, 0,0,0)$, and $T^{\alpha \beta}$ is the stress-energy tensor for a perfect fluid (see section 3 ).

Four elliptic constraint equations, which are usually referred to as Hamiltonian and momentum constraints,

$$
\begin{aligned}
& \mathcal{H} \equiv R^{(3)}+K^{2}-K_{i j} K^{i j}-16 \pi \rho_{\mathrm{ADM}}=0 \\
& \mathcal{M}^{i} \equiv D_{j}\left(K^{i j}-\gamma^{i j} K\right)-8 \pi S^{i}=0
\end{aligned}
$$

should be satisfied within each spacelike slice. Here $R^{(3)}=R_{i j} \gamma^{i j}$ is the Ricci scalar on a 3D timeslice. Additional constraints are given by

$$
\operatorname{det} \tilde{\gamma}_{i j}=1, \quad \operatorname{tr} \tilde{A}_{i j}=0, \quad \tilde{\Gamma}^{i}=\tilde{\gamma}^{j k} \tilde{\Gamma}_{j k}^{i},
$$


with the last two equations of (12) being enforced algebraically. The remaining constraint in (12), and the constraints $\mathcal{H}$ and $\mathcal{M}^{i}$ are not actively enforced and can be used as monitors of the accuracy of our numerical solution.

We specify the gauges in terms of the standard ADM lapse function, $\alpha$, and shift vector, $\beta^{a}$ [18]. We evolve the lapse according to the ' $1+\log$ ' slicing condition [19]:

$$
\partial_{t} \alpha-\beta^{i} \partial_{i} \alpha=-2 \alpha\left(K-K_{0}\right)
$$

where $K_{0}$ is the initial value of the trace of the extrinsic curvature and equals zero for the maximally sliced initial data we consider here. The code uses a hyperbolic $\tilde{\Gamma}$-driver condition [20],

$$
\begin{aligned}
& \partial_{t} \beta^{i}-\beta^{j} \partial_{j} \beta^{i}=\frac{3}{4} \alpha B^{i}, \\
& \partial_{t} B^{i}-\beta^{j} \partial_{j} B^{i}=\partial_{t} \tilde{\Gamma}^{i}-\beta^{j} \partial_{j} \tilde{\Gamma}^{i}-\eta B^{i},
\end{aligned}
$$

where $\eta$ is a parameter which acts as a damping coefficient (see the discussion in [21]).

Two routes are possible when solving numerically the Einstein equations in axisymmetric spacetimes. One route consists in using coordinates that exploit the symmetry and enforce its preservation already at a mathematical level, such as cylindrical coordinates. This advantage is counterbalanced by the fact that such coordinates are usually singular somewhere (e.g., on the axis for cylindrical coordinates) and that regularization conditions are therefore necessary (see [22, 23] and the references therein for a recent discussion).

The second route consists, instead, of using Cartesian coordinates and exploiting the fact that these coincide with the cylindrical ones in one plane, namely the $(x, z)$ plane (for concreteness we will assume hereafter that the Cartesian and the cylindrical $z$-axes coincide). The chief advantages of this approach, which is usually referred to as the cartoon method [1], are the absence of the need of regularization conditions and the easiness of implementation, through a simple dimensional reduction from fully 3D codes in Cartesian coordinates. However, these advantages are counterbalanced by at least two disadvantages. The first one is that the method still essentially requires the use of a 3D domain covered with Cartesian coordinates, although one of the three dimensions, namely the $y$-direction, has a very small extent. The second one is that, in order to compute the spatial derivatives in the $y$-direction appearing in the Einstein equations, a number of high-order interpolations onto the $x$-axis are necessary (see the discussion below), and these can amount to a significant portion of the time spent for each evolution to the new timelevel. In practice, the spatial derivatives in the $y$-direction are computed exploiting the fact that all quantities are constant on cylinders, and thus the value of a variable $\Psi$ at a generic position $(x, y, z)$ off the $(x, z)$ plane can be computed from the corresponding value $\Psi(\tilde{x}, 0, \tilde{z})$ on the $(x, z)$ plane, where

$$
\tilde{x}=(x+y)^{1 / 2}, \quad \tilde{z}=z .
$$

Clearly, since the solution of the evolution equations is computed only on the $(x, z)$ plane, interpolations (with truncation errors smaller than that of the finite-difference operators) are needed at all the positions $(\tilde{x}, y=0, \tilde{z})$.

Overall the cartoon method represents the choice for many codes, and it has been implemented with success in many applications, e.g., $[1-3,5-8,24]$ to cite a few.

\section{Evolution of the relativistic hydrodynamics equations}

An important feature of multidimensional non-vacuum numerical-relativity codes that solve the coupled Einstein-hydrodynamics equations in Cartesian coordinates is the adoption of a 
conservative formulation of the hydrodynamics equations [25, 26]. In such a formulation, the set of conservation equations for the stress-energy tensor $T^{\mu \nu}$ and for the matter current density $J^{\mu}$, that is

$$
\begin{aligned}
& \nabla_{\mu} J^{\mu}=0, \\
& \nabla_{\mu} T^{\mu \nu}=0,
\end{aligned}
$$

is written in a hyperbolic, first-order 'flux-conservative' form of the type [27]:

$$
\partial_{t} \mathbf{q}+\partial_{i} \mathbf{f}^{(i)}(\mathbf{q})=\mathbf{s}(\mathbf{q})
$$

where $\mathbf{f}^{(i)}(\mathbf{q})$ and $\mathbf{s}(\mathbf{q})$ are the flux vectors and source terms, respectively [28]. Note that the right-hand side (the source terms) depends only on the metric, on its first derivatives and on the stress-energy tensor. Furthermore, while the system (19) is not strictly hyperbolic, strong hyperbolicity is recovered in a flat spacetime, where $\mathbf{s}(\mathbf{q})=0$.

As shown in [26], in order to write the system (17)-(18) in the form of system (19), the primitive hydrodynamical variables (i.e., the rest-mass density $\rho$, the pressure $p$ measured in the rest frame of the fluid, the fluid 3-velocity, $v^{i}$, measured by a local zero-angular momentum observer, the specific internal energy $\epsilon$ and the Lorentz factor $W$ ) are mapped to the so-called conserved variables $\mathbf{q} \equiv\left(D, S^{i}, \tau\right)$ via the relations

$D \equiv \sqrt{\gamma} \rho W, \quad S^{i} \equiv \sqrt{\gamma} \rho h W^{2} v^{i}, \quad \tau \equiv \sqrt{\gamma}\left(\rho h W^{2}-p\right)-D$,

where $h \equiv 1+\epsilon+p / \rho$ is the specific enthalpy, and $W \equiv\left(1-\gamma_{i j} v^{i} v^{j}\right)^{-1 / 2}$.

The advantage of a flux-conservative formulation is that it allows us to use high-resolution shock-capturing (HRSC) schemes, which are based on Riemann solvers, and which are essential for a correct representation of shocks. This is particularly important in astrophysical simulations, where large shocks are expected. In this approach, all variables $\mathbf{q}$ are represented on the numerical grid by cell-integral averages. The function is then reconstructed within each cell, usually through piecewise polynomials, in a way that preserves the conservation of the variables q. This gives two values at each cell boundary, which are then used as initial data for the (approximate) Riemann problem, whose solution gives the flux through the cell boundary.

As in the Whisky code, the evolution equations are here integrated in time using the method of line [29], which reduces the partial differential equations (19) to a set of ordinary differential equations that can be evolved using standard numerical methods, such as RungeKutta or the iterative Cranck-Nicolson schemes [30, 31]. Furthermore, the Whisky2D code implements several reconstruction methods, such as total-variation-diminishing (TVD) methods, essentially-non-oscillatory (ENO) methods [32] and the piecewise-parabolic method (PPM) [33]. Also, a variety of approximate Riemann solvers can be used, starting from the Harten-Lax-van Leer-Einfeldt (HLLE) solver [34], over to the Roe solver [35] and the Marquina flux formula [36] (see [10,11] for a more detailed discussion).

The ability of properly evolving large gradients moving at relativistic speeds represents one of the main motivations that make this formulation the choice for all of the present 3D numerical-relativity codes solving the relativistic hydrodynamics equations on Eulerian grids (see [37-39] for some of the most recent examples and [40] for an alternative Lagrangian method). However, when considered within the axisymmetric approach used here, the use of a flux-conservative formulation in Cartesian coordinates may suffer from a potentially very serious disadvantage. In fact, the interpolations required by the cartoon method may be highly inaccurate when discontinuities in the fluid variables appear. To confront this problem, Shibata [2] has made the useful suggestion of writing the relativistic hydrodynamics equations in cylindrical coordinates, while keeping the solution of the Einstein equations in Cartesian 
coordinates. This approach has the obvious advantage that it does not require interpolation and that it exploits, at the mathematical level, the symmetries of the system, thus guaranteeing a better conservation of mass and angular momentum. However, the use of cylindrical coordinates for the evolution of the fluid variables also comes with an undesirable property: the coordinates are degenerate at the symmetry axis, and the equations are no longer free of singularities. As we will comment in the following section, this drawback can be compensated through a suitable formulation of the equations and a proper setup of the numerical grid.

\subsection{A new formulation of the hydrodynamics equations}

As mentioned in the previous section, following [3], we write the relativistic hydrodynamics equations (17)-(18) in a first-order form in space and time using cylindrical coordinates $(r, \phi, z)$. However, as an important difference from the approach suggested in [3], we do not introduce source terms that contain coordinate singularities. Rather, we redefine the conserved quantities in such a way to remove the singular terms, which are the largest source of truncation error, also when evaluated far from the axis.

We illustrate our approach by using as a representative example the continuity equation. This is the simplest of the five hydrodynamical equations, but already contains all the basic elements necessary to illustrate the new formulation. We start by using the definitions for the conserved variables (20) to write equation (17) generically as

$$
\partial_{t}(\sqrt{\gamma} \rho W)+\partial_{i}\left[\sqrt{\gamma} \rho W\left(\alpha v^{i}-\beta^{i}\right)\right]=0,
$$

which in cylindrical coordinates takes the form

$$
\partial_{t}(\sqrt{\tilde{\gamma}} \rho W)+\partial_{r}\left[\sqrt{\tilde{\gamma}} \rho W\left(\alpha v^{r}-\beta^{r}\right)\right]+\partial_{z}\left[\sqrt{\tilde{\gamma}} \rho W\left(\alpha v^{z}-\beta^{z}\right)\right]=0,
$$

where $\sqrt{\tilde{\gamma}}$ is the determinant of the 3-metric in cylindrical coordinates, and where we have enforced the condition of axisymmetry $\partial_{\phi}=0$. Because any $\phi$-constant plane in cylindrical coordinates can be mapped into the $(x, z)$ plane in Cartesian coordinates, we consider equation (22) as expressed in Cartesian coordinates and restricted to the $y=0$ plane, i.e.,

$$
\partial_{t}(x D)+\partial_{x}\left[x D\left(\alpha v^{x}-\beta^{x}\right)\right]+\partial_{z}\left[x D\left(\alpha v^{z}-\beta^{z}\right)\right]=0,
$$

where we have exploited the fact that for any vector of components $A^{i}$ on this plane $A^{r}=A^{x}, A^{\phi}=A^{y}$ and $\tilde{\gamma}=x^{2} \gamma$, with $\gamma$ being the determinant of the 3-metric in Cartesian coordinates. Equation (23) represents the prototype of the formulation proposed here, which we will refer to hereafter as the ' $n e w$ ' formulation to contrast it with the formulation adopted so far, e.g., in [3], for the solution of the relativistic hydrodynamics equations in axisymmetry and in Cartesian coordinates. The only, but important, difference with respect to the 'standard' formulation is that in the latter the derivative in the $x$-direction is written out explicitly, and becomes part of the source term $\mathbf{s}(\mathbf{q})$, i.e.,

$$
\partial_{t}(D)+\partial_{x}\left[D\left(\alpha v^{x}-\beta^{x}\right)\right]+\partial_{z}\left[D\left(\alpha v^{z}-\beta^{z}\right)\right]=-\frac{D\left(\alpha v^{x}-\beta^{x}\right)}{x} .
$$

Even though the right-hand side of equation (24) is never evaluated at $x=0$ (because no grid points are located at $x=0$ ), both the numerator and the denominator of the right-hand side of equation (24) are very small for $x \simeq 0$, so that small round-off errors in the evaluation of the right-hand side can increase the overall truncation error. Stated differently, the right-hand side of equation (24) becomes stiff for $x \simeq 0$ and this opens door to the problems encountered in the numerical solution of hyperbolic equations with stiff source terms [41].

What was done for the continuity equation (23) can be extended to the other hydrodynamics equations which, for the conservation of momentum in the $x$-and $z$-directions, 
take the form

$$
\begin{gathered}
\frac{1}{\alpha x \sqrt{\gamma}}\left\{\partial_{t}\left(x S_{A}\right)+\partial_{x}\left[x\left(S_{A}\left(\alpha v^{x}-\beta^{x}\right)+\alpha \sqrt{\gamma} p \delta_{A}^{x}\right)\right]+\partial_{z}\left[x\left(S_{A}\left(\alpha v^{z}-\beta^{z}\right)+\alpha \sqrt{\gamma} p \delta_{A}^{z}\right)\right]\right\} \\
=\left[T^{00}\left(\frac{1}{2} \beta^{l} \beta^{m} \partial_{A} \gamma_{l m}-\alpha \partial_{A} \alpha\right)+T^{0 i} \beta^{l} \partial_{A} \gamma_{i l}+T_{i}^{0} \partial_{A} \beta^{i}+\frac{1}{2} T^{l m} \partial_{A} \gamma_{l m}\right],
\end{gathered}
$$

with $A=x, z$. Similarly, the evolution of the conserved angular momentum, $S_{\phi}=x S_{y}$, is expressed as

$$
\frac{1}{\alpha x \sqrt{\gamma}}\left\{\partial_{t}\left(x^{2} S_{y}\right)+\partial_{x}\left[x^{2} S_{y}\left(\alpha v^{x}-\beta^{x}\right)\right]+\partial_{z}\left[x^{2} S_{y}\left(\alpha v^{z}-\beta^{z}\right)\right]\right\}=0,
$$

while the equation of the energy conservation is given by

$$
\begin{aligned}
\frac{1}{\alpha x \sqrt{\gamma}}\left\{\partial_{t}(x \tau)\right. & \left.+\partial_{x}\left[x\left(\tau\left(\alpha v^{x}-\beta^{x}\right)+p v^{x}\right)\right]+\partial_{z}\left[x\left(\tau\left(\alpha v^{z}-\beta^{z}\right)+p v^{z}\right)\right]\right\} \\
& =T^{00}\left(\beta^{i} \beta^{j} K_{i j}-\beta^{i} \partial_{i} \alpha\right)+T^{0 i}\left(-\partial_{i} \alpha+2 \beta^{j} K_{i j}\right)+T^{i j} K_{i j} .
\end{aligned}
$$

The changes made to the formulation are rather simple but, as we will show in section 4 , these can produce significant improvements on the overall accuracy of the simulations with a truncation error at least one order of magnitude smaller for all of the tests considered. Because of its simplicity, the changes in the new formulation of the equations can be implemented straightforwardly in codes written using the standard formulation.

Finally, we note that both equations (23) and (24) are written in a flux-conservative form in the sense that the source term does not contain first-order spatial derivatives of the conserved variables. More precisely, equation (23) is written in a flux-conservative form, while equation (24) is written in a 'flux-balanced' form, as it is typical for flux-conservative equations written in curvilinear coordinates [27]. The same is true also for equations (25)(27) and for the corresponding equations presented in [3], which are incorrectly classified as non-flux conservative.

\subsection{Equation of state}

In whatever coordinate system they are written, the system of hydrodynamics equations can be closed only after specifying an additional equation, the equation of state (EOS), which relates the pressure to the rest-mass density and to the energy density. The code has been written to use any EOS, but all the tests so far have been performed using either an (isentropic) polytropic EOS,

$$
\begin{aligned}
& p=K \rho^{\Gamma}, \\
& e=\rho+\frac{p}{\Gamma-1},
\end{aligned}
$$

or an 'ideal-fluid' EOS,

$$
p=(\Gamma-1) \rho \epsilon
$$

Here, $e$ is the energy density in the rest frame of the fluid, $K$ the polytropic constant (not to be confused with the trace of the extrinsic curvature defined earlier), and $\Gamma$ is the adiabatic exponent. In the case of the polytropic $\operatorname{EOS~(28),~} \Gamma=1+1 / N$, where $N$ is the polytropic index, and the evolution equation for $\tau$ does not need to be solved. In the case of the idealfluid EOS (30), on the other hand, non-isentropic changes can take place in the fluid, and the evolution equation for $\tau$ needs to be solved. Note that the polytropic EOS (28) is isentropic and thus does not allow for the formation of physical shocks, in which entropy (and internal energy) can be increased locally (shock heating). 
Table 1. Equilibrium properties of the initial stellar models. The different columns refer respectively to the ratio of the polar-to-equatorial-coordinate radii $r_{p} / r_{e}$, the central rest-mass density $\rho_{c}$, the gravitational mass $M$, the rest mass $M_{0}$, the circumferential equatorial radius $R_{e}$, the angular velocity $\Omega$, the maximum angular velocity for a star of the same rest mass $\Omega_{K}$, the ratio $J / M^{2}$ where $J$ is the angular momentum, and the ratio of rotational kinetic energy to gravitational binding energy $T /|W|$. All models have been computed with a polytropic EOS with $K=100$ and $\Gamma=2$.

\begin{tabular}{|c|c|c|c|c|c|c|c|c|c|}
\hline & $r_{p} / r_{e}$ & $\begin{array}{l}\rho_{c} \\
\left(\times 10^{-3}\right)\end{array}$ & $\begin{array}{l}M \\
\left(M_{\odot}\right)\end{array}$ & $\begin{array}{l}M_{0} \\
\left(M_{\odot}\right)\end{array}$ & $R_{e}$ & $\Omega$ & $\begin{array}{l}\Omega_{K} \\
\left(\times 10^{-2}\right)\end{array}$ & $J / M^{2}$ & $T /|W|$ \\
\hline A & 1.00 & 1.28 & 1.400 & 1.506 & 9.586 & 0.000 & 3.987 & 0.000 & 0.000 \\
\hline B & 0.67 & 1.28 & 1.651 & 1.786 & 12.042 & 0.253 & 3.108 & 0.594 & 0.081 \\
\hline
\end{tabular}

\section{Numerical tests}

In order to test the stability properties of the new formulation and compare its accuracy with that of the formulation first presented in [3] and then used, among others, in [5, 6, 8, 42, 43], we have implemented both of them in Whisky2D. In the following sections, we test them against well-known smooth solutions in curved but static spacetimes, or in curved and fully dynamical spacetimes, and, finally, against discontinuous solutions in flat spacetimes.

The initial data, in particular, have been produced as the solution of the Einstein equations for axisymmetric and stationary stellar configurations [44], using the EOS (28) with $\Gamma=2$ and polytropic constant $K=100$, in order to produce stellar models, which are, at least qualitatively, representative of what is expected from observations of neutron stars. Our attention has been restricted to two illustrative models representing a nonrotating star and a rapidly rotating star having equatorial and polar (coordinate) radii in a ratio $r_{p} / r_{e}=0.67$. The relevant properties of these stellar models are reported in table 1.

All the numerical results presented hereafter have been obtained with the following fiducial numerical set-up: the reconstruction of the values at the boundaries of the computational cells is made using the PPM method [33], while the HLLE algorithm is used as an approximate Riemann solver [34]. The lapse function is evolved with the ' $1+\log$ ' slicing condition given by equation (13), while the shift is evolved using a version of the hyperbolic $\tilde{\Gamma}$-driver condition (14) in which the advection terms for the variables $\beta^{i}, B^{i}$ and $\tilde{\Gamma}^{i}$ are set to zero. The time evolution is made with a method-of-line approach [29] and a third-order Runge-Kutta integration scheme (our CFL factor is usually chosen between 0.3 and 0.5). A third-order Lagrangian interpolation is adopted to implement the cartoon method. For the matter variables we use 'Dirichlet' boundary conditions (i.e., the solution at the outer boundary is always kept to be the initial one), while for the field variables we adopt outgoing Sommerfeld boundary conditions.

We typically present results at four different resolutions: $\bar{h}=0.4 M, \bar{h} / 2, \bar{h} / 4,3 \bar{h} / 16$ and $\bar{h} / 8$, which correspond to about $25,50,100,133$ and 200 points across the stellar radius, respectively. The computational domain extends to $20 M$ both in the $x$ - and $z$-directions, and a reflection symmetry is applied across the equatorial (i.e., $z=0$ ) plane. Finally, we remark that in contrast to the interesting analysis of [45], we could not find signs of numerical instabilities when using the above numerical prescriptions for either of the two formulations considered.

\subsection{Oscillating neutron stars: fixed spacetime}

The first set of tests, we discuss, has been carried out by simulating relativistic polytropic stars in equilibrium and in a fixed spacetime (i.e., in the Cowling approximation). In this case, the 

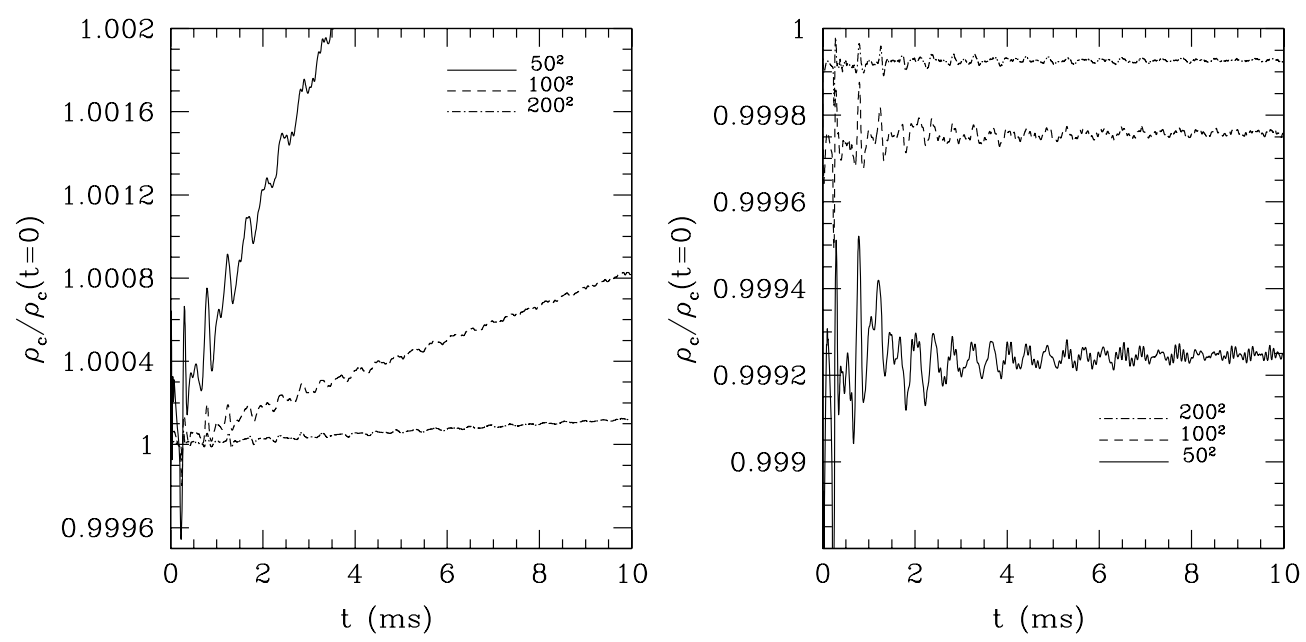

Figure 1. Evolution of the central rest-mass density for rapidly rotating stars (model B in table 1) evolved within the Cowling approximation. The left panel refers to the use of the standard formulation, while the right one to the new formulation. Note the different scales in the two panels and note that in both cases the amplitude of the oscillations decreases with increasing resolution, while keeping the same phase.

Einstein equations are not evolved and the truncation error is in general smaller because it is produced uniquely from the evolution of the hydrodynamics equations.

Although the stars are in equilibrium, oscillations are triggered by the first-order truncation error at the centre and the surface of the star (our hydrodynamical evolution schemes are only first order at local extrema). Both the amplitude of the oscillations and the rate of the secular change in their amplitude converge to zero at nearly second order with increasing grid resolution $[46,47]$. The genuine dynamics produced by the truncation error can then be studied either when the spacetime is held fixed (i.e., in the Cowling approximation) or when the spacetime is evolved through the solution of the Einstein equations. This is shown in figure 1, which reports the evolution of the central rest-mass density for rapidly rotating stars (model B in table 1) evolved within the Cowling approximation. The left panel refers to the standard formulation, while the right one to the new formulation. Note that in both cases the amplitude of the oscillations decreases at roughly second order with increasing resolution, while keeping the same phase. This is a clear signature that the oscillations corresponds to proper eigenmodes of the simulated star. However, the difference of the secular evolution between the standard formulation and the new one is rather remarkable. The latter, in fact, is much more accurate, and the well-known secular increase in the central density is essentially absent in the new formulation.

Quantities that are particularly useful to assess the accuracy of the two formulations are the rest mass and the angular momentum which we compute as [48]

$M_{0}=2 \pi \int_{V_{*}} \sqrt{\gamma} \rho W x \mathrm{~d} x \mathrm{~d} z$

$J_{z}=2 \pi \epsilon_{z j}^{k} \int_{V}\left(\frac{1}{8 \pi} \tilde{A}_{k}^{j}+x^{j} S_{k}+\frac{1}{12 \pi} x^{j} K_{, k}-\frac{1}{16 \pi} x^{j} \tilde{\gamma}_{, k}^{l m} \tilde{A}_{l m}\right) \mathrm{e}^{6 \Phi} x \mathrm{~d} x \mathrm{~d} z$

where $V_{*}$ is the coordinate volume occupied by the star, and $V$ is coordinate volume of the computational domain. 

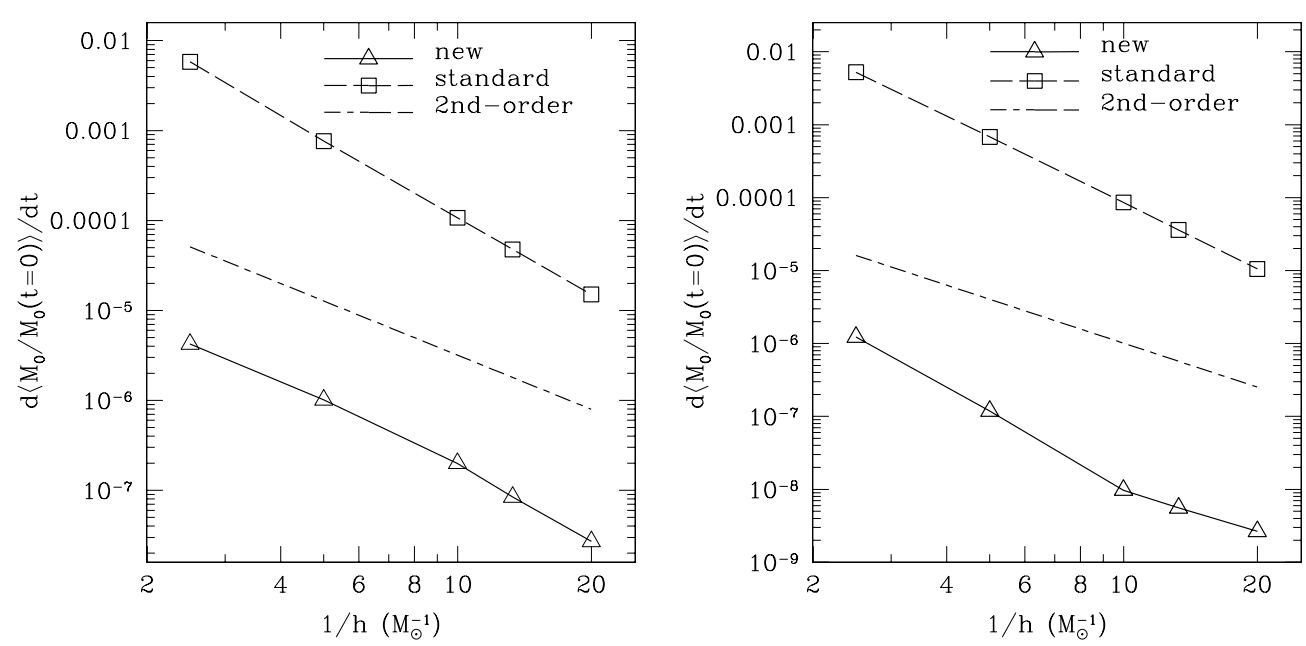

Figure 2. Time derivative of the average of the rest mass $M_{0}$, normalized to the initial value $M_{0}(t=0)$, for evolutions in a fixed spacetime (Cowling approximation). The average $\mathrm{d}\left\langle M_{0} / M_{0}(t=0)\right\rangle / \mathrm{d} t$ is computed between the initial value and a time $t=25 \mathrm{~ms}$, corresponding to about 30 oscillations. The left panel refers to a nonrotating star (model A in table 1), while the right panel to a rapidly rotating star (model B in table 1). Indicated with squares are the numerical values obtained with the standard formulation of the hydrodynamics equations, while triangles are used for the new one. Also indicated with a dot-dashed line is the slope for a second-order convergence rate.

Figure 2 shows the dependence on the inverse of the resolution of the error in the conservation of the rest mass for a nonrotating model as computed in the Cowling approximation (left panel) or in a fully dynamical simulation (right panel). Since the evolution of the rest mass shows, in addition to a secular evolution, small oscillations (i.e., of $\sim 3 \times 10^{-9}$ for the highest resolution and of $\sim 3 \times 10^{-6}$ for the lowest resolution) the calculation of the rest mass at a given time can be somewhat ambiguous. To tackle this problem and to avoid the measurement being spoiled by the oscillations, we perform a linear fit of the evolution of $M_{0}$, normalized to the initial value $M_{0}(t=0)$, between the initial value and a time $t=25 \mathrm{~ms}$ (corresponding to about 30 oscillations) and then compute the time average derivative $\mathrm{d}\left\langle M_{0} / M_{0}(t=0)\right\rangle / \mathrm{d} t$. Figure 2, in particular, reports in a logarithmic scale, $\mathrm{d}\left\langle M_{0} / M_{0}(t=0)\right\rangle / \mathrm{d} t$, as a function of the inverse of the resolution $h$. Indicated with squares are the numerical values obtained with the standard formulation of the hydrodynamics equations, while triangles are used for the new one. Also indicated with a long-short-dashed line is the slope for a second-order convergence rate.

Note that although we use a third-order method for the reconstruction (namely, PPM), we do not expect third-order convergence. This is also due to the fact that the reconstruction schemes are only first-order accurate at local extrema (i.e., at the centre and at the surface of the star), thus increasing the overall truncation error. Similar estimates were obtained also using the Whisky code in 3D Cartesian coordinates [10, 11].

Clearly both the new and the standard methods provide a convergence rate which is close to two. However, and this is the most important result of this work, the new method yields a truncation error which is several orders of magnitude smaller than the old one. More specifically, in the case of the rest mass, the conservation is more accurate of about four orders of magnitude. We believe that this is essentially due to the rewriting of the source terms in the flux-conservative formulation, which in the new formulation does not have any coordinate-singular term (i.e., $\propto 1 / x)$. 


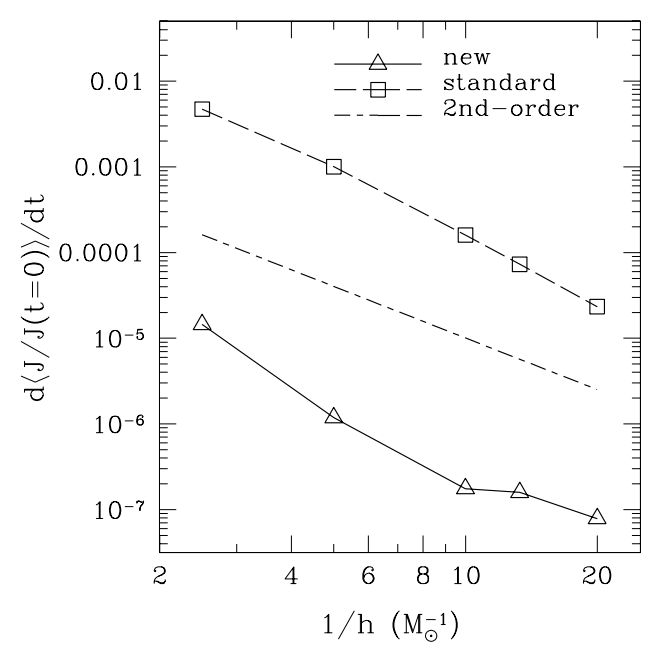

Figure 3. Time derivative of the average of the angular momentum normalized to the initial value $\mathrm{d}\langle J / J(t=0)\rangle / \mathrm{d} t$ (cf figure 2 ) for a rapidly rotating star (model B of table 1). Indicated with squares are the numerical values obtained with the standard formulation of the hydrodynamics equations, while triangles are used for the new one; a dot-dashed line is the slope for a second-order convergence rate.

Note also that, because the new formulation is intrinsically more accurate, it also suffers more easily from the contamination of errors which are not directly related to the finitedifference operators. (That made in the calculation of the integral (32) is a relevant example, but it is not the only one.) This may be the reason why, in general, at lower resolutions the new formulation has a convergence rate, which is not exactly two and appears over-convergent (see the right panel of figure 2). However, as the resolution is increased and the finite-difference errors become the dominant ones, a clearer trend in the convergence rate is recovered.

Another way of measuring the accuracy of the two formulations is via the comparison of the evolution of the angular momentum. While this quantity is conserved to machine precision in the case of a nonrotating star, this does not happen for rotating stars, and the error can be of a few percent in the case of very low resolution and of a very rapidly rotating star. This is shown in figure 3 for the stellar model B of table 1 , and it reports in a logarithmic scale the time derivative of the average of the angular momentum $J$ normalized to the initial value $J(t=0)$. In analogy with figure 2 , in order to remove the small-scale oscillations, we first perform a linear fit of the evolution of $J$ between the initial value and a time $t=25 \mathrm{~ms}$, and take the coefficient of the fit as the time derivative of the angular momentum: $\mathrm{d}\langle J / J(t=0)\rangle / \mathrm{d} t$. Indicated with squares are the numerical values obtained with the standard formulation of the hydrodynamics equations, while triangles are used for the new one; a dot-dashed line shows the slope for a second-order convergence rate.

It is simple to recognize from figure 3 that also for the angular momentum conservation the new formulation yields a truncation error which is two or more orders of magnitude smaller, with a clear second-order convergence being recovered at sufficiently high resolution.

\subsection{Oscillating neutron stars: dynamical spacetime}

Also the second set of tests we discuss is based on the evolution of relativistic polytropic stars in equilibrium, but now the evolution is performed in a dynamical spacetime, thus with 

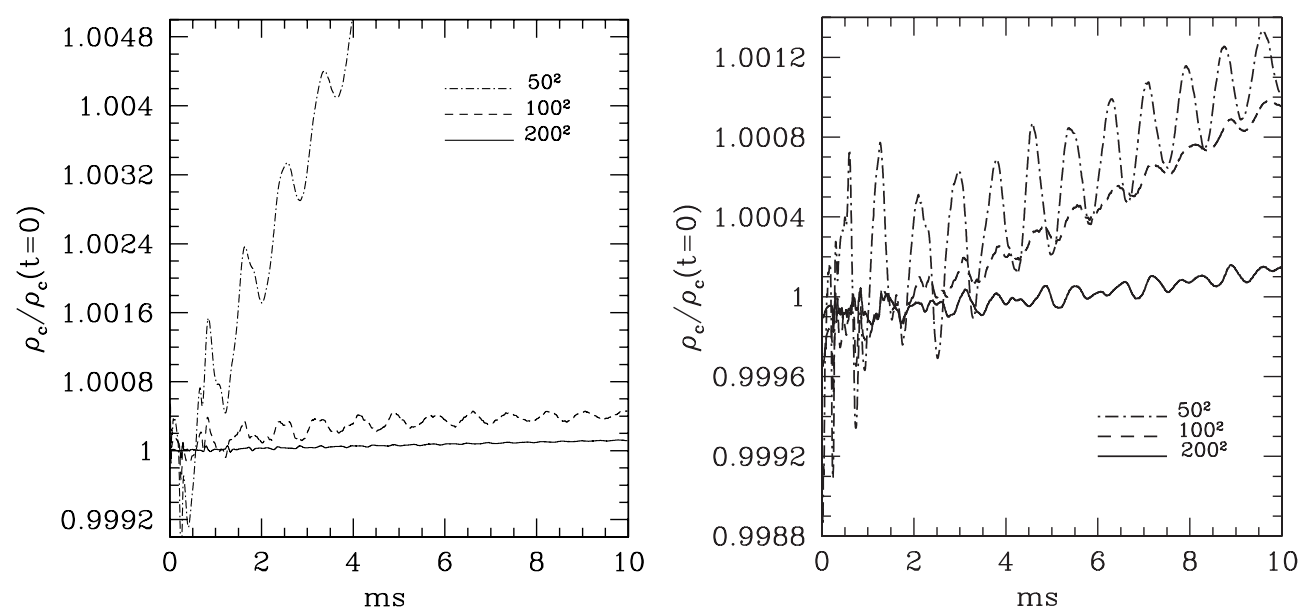

Figure 4. The same as in figure 1, but for a full-spacetime evolution. The left panel refers to the standard formulation, while the right one to the new formulation. Note the different scale between the two panels.
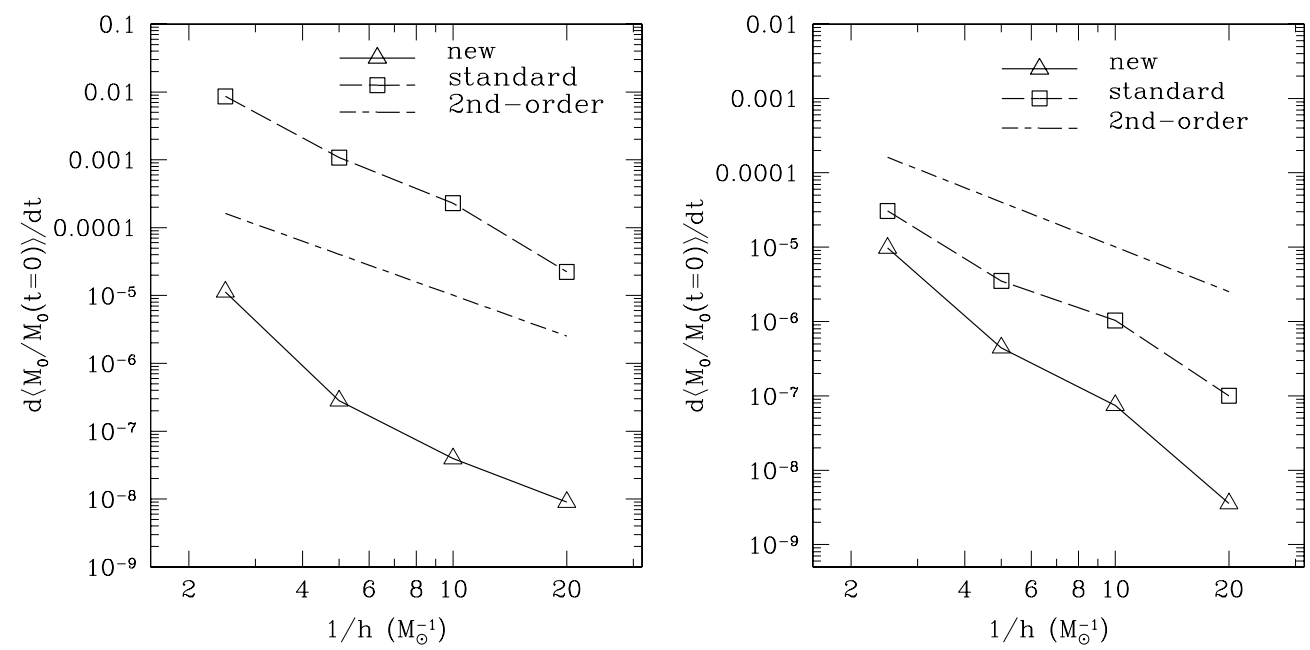

Figure 5. The same as in figure 2, but for full-spacetime evolutions. The left panel refers to a nonrotating star (model A in table 1), while the right panel to a rapidly rotating star (model B in table 1).

the coupling of Einstein and hydrodynamics equations. The truncation error in this case is given by the truncation error coming from the solution of both the field equations and the hydrodynamics equations. The results of our calculations are summarized in figures 4-6, which represent the equivalents of figures 1-3 for full-spacetime evolutions. Because the results are self-explanatory and qualitatively similar to those discussed for the evolutions with fixed spacetimes, we will comment on them only briefly.

In particular, figures 5 and 6 highlight that while the overall truncation error in dynamical spacetimes is essentially unchanged for the standard formulation, it has increased in the case of the new formulation. This is particularly evident at very low resolutions, where the new 


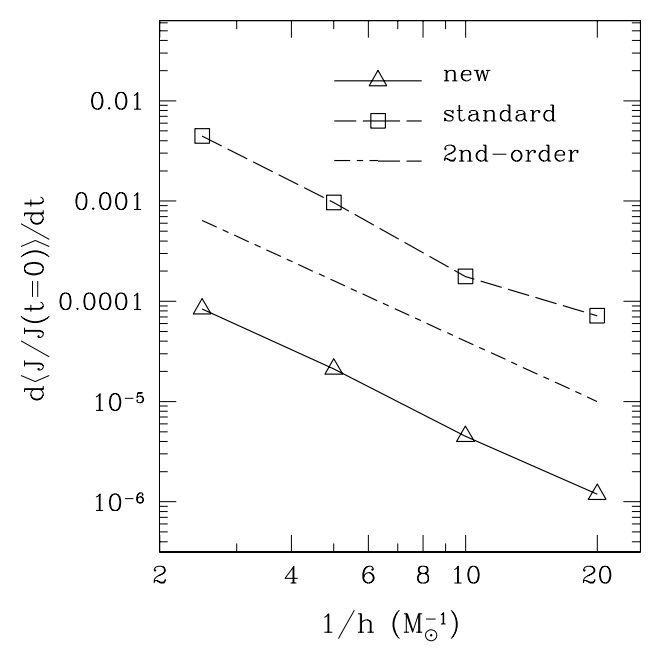

Figure 6. The same as figure 3, but for a rapidly rotating star evolved in a dynamical spacetime.

formulation seems to be hyper-convergent. However, despite a truncation error which is larger than that for fixed spacetimes, the figures also indicate that the new formulation does represent a considerable improvement over the standard one, and that its truncation error is at least two orders of magnitude smaller. Most importantly, the conservation properties of the numerical scheme have greatly improved, and the secular increase in the rest mass is also considerably suppressed. This is clearly shown in figure 4, where the secular increase is suppressed almost quadratically with resolution. More precisely, for both approaches the growth rate of the central rest-mass density for the coarse resolution is $\sim 12$ times larger than the corresponding one for the high resolution. However, at the highest resolution, the growth rate for the standard formulation is $\sim 10$ times larger than that of the new formulation.

\subsection{Calculation of the eigenfrequencies}

As mentioned in the previous section, although in equilibrium, the simulated stars undergo oscillations which are triggered by the nonzero truncation error. It is possible to consider these oscillations not as a numerical nuisance, on the contrary, it is possible to exploit them to perform a check on the consistency of a full nonlinear evolution with a small perturbation (the truncation error) with the predictions of perturbation theory $[46,47]$. Furthermore, when used in conjunction with highly accurate codes, these oscillations can provide important information on the stellar oscillations within regimes, such as those of very rapid or differential rotation, which are not yet accessible via perturbative calculations [49].

In this section, we use such oscillations, and, in particular, the fundamental $\ell=0$ quasiradial $F$-mode, to compare the accuracy of the two formulations against the perturbative predictions. This is summarized in figure 7, which reports the power spectral density (in arbitrary units) of the maximum rest-mass density evolution (cf, figures 1 and 3 ) in the new and standard formulations (solid and dashed lines, respectively). The simulations are relative to a nonrotating star (model $\mathrm{A}$ in table 1) with the left panel referring to an evolution with a fixed spacetime, while the right one to an evolution with a dynamical spacetime. The specific spectra shown are calculated from the simulations at the highest resolution and cover an interval of $25 \mathrm{~ms}$. It is quite apparent that the two formulations yield spectra which are extremely 

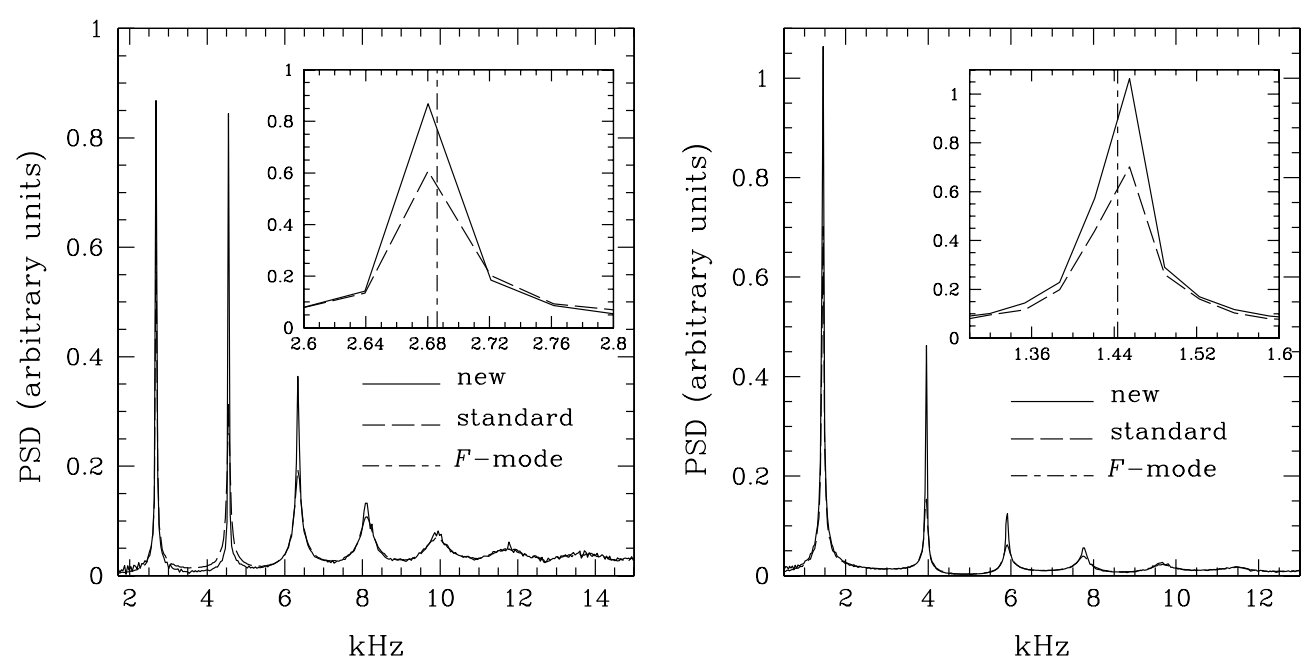

Figure 7. Power spectral density (in arbitrary units) of the maximum rest-mass density evolution in the new and standard formulations (solid and dashed lines, respectively). The simulations are relative to a nonrotating star (model A in table 1) with the left panel referring to an evolution with a fixed spacetime and the right one to an evolution with a dynamical spacetime. The spectra are calculated from the simulations at the highest resolution and cover $25 \mathrm{~ms}$ of evolution. For both panels the insets show a magnification of the spectra near the $F$-mode and the comparison with the perturbative estimate as calculated with the numerical code described in [50].

similar, with a prominent $F$-mode at about $2.7 \mathrm{kHz}$ and $1.4 \mathrm{kHz}$ for the fixed and dynamical spacetime evolutions, respectively. The spectra also show the expected quasi-radial overtones at roughly multiple integers of the $F$-mode, the first of which has a comparable power in the case of Cowling evolution, while it is reduced of about $50 \%$ in the full-spacetime evolution. Indeed, the spectra in the two formulations are so similar that it is necessary to concentrate on the features of the $F$-mode to appreciate the small differences. These are shown in the insets of the two panels which report, besides a magnification of the spectra near the $F$-mode, also the perturbative estimate $F_{\text {pert }}$, as calculated with the perturbative code described in [50].

To provide a more quantitative assessment of the accuracy with which the two formulations reproduce the perturbative result we have computed the eigenfrequency of the $F$-mode, which we indicate as $F_{\text {num }}$, by performing a Lorentzian fit to the power spectrum with a window of $0.2 \mathrm{kHz}$. We remark that it is essential to make use of a Lorentzian function for the fit as this reflects the expected functional behaviour and increases the accuracy of the fit significantly. Shown in figure 8 is the absolute value of the relative difference between the numerical and perturbative eigenfrequencies of the $F$-mode, $\left|1-F_{\text {num }} / F_{\text {pert }}\right|$ for the two formulations (solid lines for the new one and dashed lines for the standard one). The differences are computed for different resolutions with $\bar{h}=0.4 M, \bar{h} / 2$ and $\bar{h} / 4$, and refer to the nonrotating mode A of table 1 when evolved in a fixed spacetime (left panel) and in a dynamical one (right panel). Indicated with a dot-dashed line is the slope for a second-order convergence rate. This helps to see that both formulations yield an almost second-order convergent measure of the eigenfrequencies of the $F$-mode, with the new formulation having a truncation error which is always smaller than that coming from the standard formulation. Given the importance of an accurate measurement of the eigenfrequencies to study the mode properties of compact stars, we believe that figures 7 and 8 provide an additional evidence of the advantages of the new formulation. 

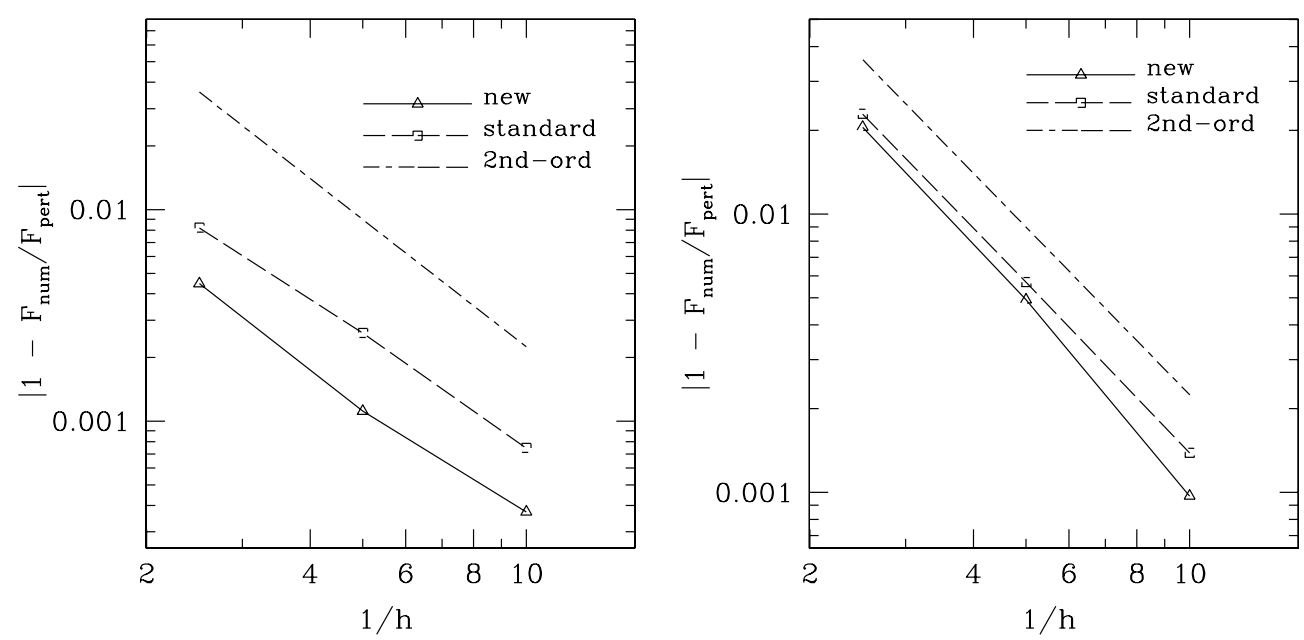

Figure 8. Relative difference between the numerical and perturbative eigenfrequencies of the $F$-mode for the two formulations (solid lines for the new one and dashed lines for the standard one). The differences are computed for different resolutions and refer to the nonrotating model A of table 1 when evolved in a fixed spacetime (left panel) and in a dynamical one (right panel). Indicated with a dot-dashed line is the slope for a second-order convergence rate.

Finally, we note that a behaviour similar to that shown in figures 7-8 has been found also for rotating stars, although in this case the comparison is possible only for evolutions within the Cowling approximation since we lack a precise perturbative estimate of the eigenfrequency for model B of table 1 for a dynamical spacetime.

\subsection{Cylindrical shock reflection}

One of the most important properties of HRSC schemes is their capability of handling the formation of discontinuities, such as shocks, which are often present and play an important role in many astrophysical scenarios. Tests involving shock formation are usually quite demanding, and codes that are not flux conservative can also show numerical instabilities or difficulties in converging to the exact solution of the problem. Since both the new and the standard formulation solve the relativistic hydrodynamics equations as written in a flux-conservative form, they are both expected to be able to correctly resolve the formation of shocks, although each with its own truncation error. In the following test, we consider one of such discontinuous flows and show that the new formulation provides a higher accuracy with respect to the standard one, stressing once again on the importance of the definition of the conserved variables.

More specifically, we consider a one-dimensional test, first proposed by [51], describing the reflection of a shock wave in cylindrical coordinates. The initial data consist of a pressureless gas with uniform density $\rho_{0}=1.0$, radial velocity $v_{0}^{x}=0.999898$, corresponding to an initial Lorentz factor $W_{0}=70.0$ and an internal energy which is taken to be small and proportional to the initial Lorentz factor, i.e., $\epsilon=10^{-5}\left(W_{0}\right)$. During the evolution an idealfluid EOS (30) is used with a fixed adiabatic index, $\Gamma=4 / 3$. The symmetry condition at $x=0$ produces a compression and generates an outgoing shock in the radial direction. The analytic solution for the values of pressure, density, gas and shock velocities are given in [51]. 

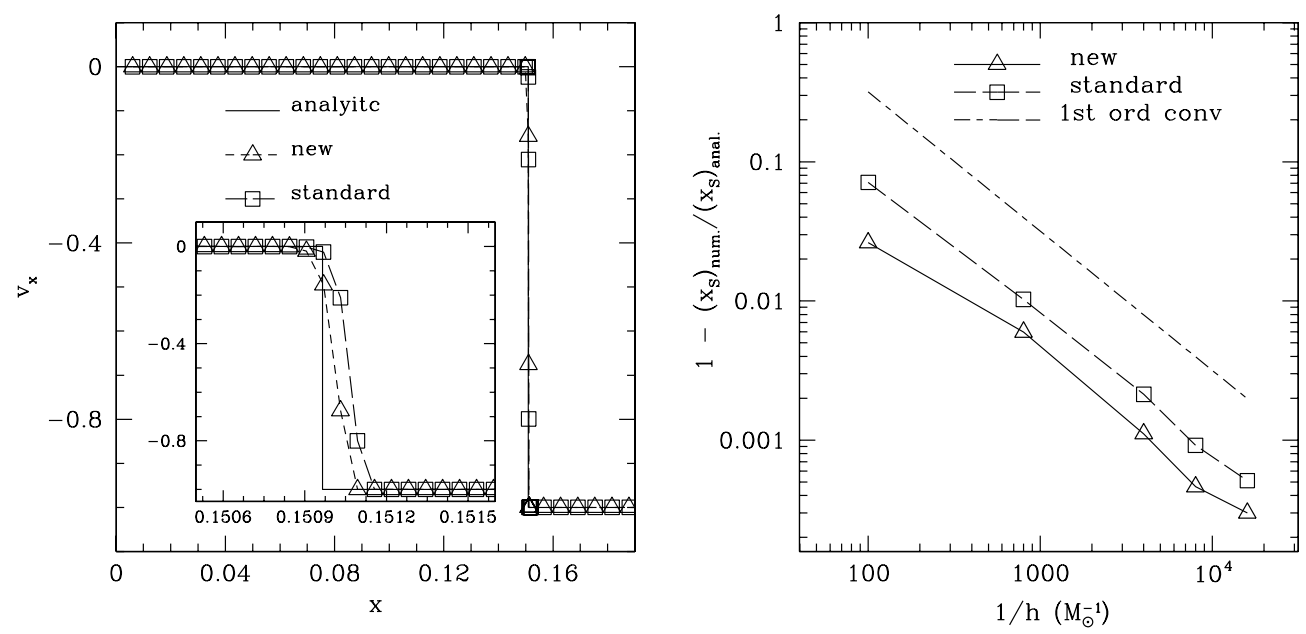

Figure 9. Left panel: comparison of the velocity profiles for the two formulations in the solution of the axisymmetric shock-tube test with a resolution of $h=6.25 \times 10^{-5} M_{\odot}$. The solid line shows the exact position after a time $\bar{t}=0.002262 \mathrm{~ms}$, while the short-dashed and the long-dashed lines represent the solutions with the new and the standard formulations, respectively. Right panel: comparison of the error in the determination of the position of the shock in the two formulations. Note the first-order convergence rate as expected for discontinuous flows.

From them, one can determine the position $x_{\mathrm{s}}$ of the shock front at any time $\bar{t}$ :

$$
x_{\mathrm{s}}=\frac{(\Gamma-1) W_{0}\left|v_{0}^{x}\right|}{W_{0}+1} \bar{t} .
$$

This can then be used to compare the accuracy of the two formulations.

In the left panel of figure 9, we show the value of the radial component of the velocity, $v_{x}$, as a function of $x$ at a time $\bar{t}=0.002262 \mathrm{~ms}$ and for a resolution of $h / M_{\odot}=6.25 \times 10^{-5}$. The solid line represents the analytic solution, the short-dashed line the numerical solution computed with the new formulation and the long-dashed line that obtained with the standard formulation. As is evident from the inset, the position of the shock is very well captured by both formulations, but the new one is closer to the exact one at this time.

To compare with the exact prediction given by expression (33), we compute the numerical position of the shock as the middle of the region where the value of the velocity moves from the pre-shock value, $v_{x}^{+}$, to the post-shock one, $v_{x}^{-}$, (in practice, we fit a straight line between the last point of the constant post-shock state and the first point of the constant pre-shock state and evaluate the position at which this function has the value, $\left.\left(v_{x}^{+}+v_{x}^{-}\right) / 2\right)$. The right panel of figure 9 shows the relative error $1-\left(x_{\mathrm{s}}\right)_{\text {num }} /\left(x_{\mathrm{s}}\right)_{\text {anal }}$ in the position of the shock at a time $\bar{t}=0.002622 \mathrm{~ms}$ and for five different resolutions: $\bar{h}=0.01 M_{\odot}, \bar{h} / 8, \bar{h} / 40, \bar{h} / 80$ and $\bar{h} / 160$. Indicated with a dashed line is the error computed when using the standard formulation, while indicated with a solid line is the error coming from the new formulation. Note that both formulations show a first-order convergence, as expected for HRSC schemes in the presence of a discontinuous flow, but, as for the other tests, also in this case the new formulation has a smaller truncation error. A similar behaviour is shown also by other quantities in this test, but these are not reported here.

It is useful to note that the difference between the two formulations in this test is smaller than in the previous ones, being of a factor of a few only and not of orders of magnitude. We believe that this is due in great part to the fact that, in contrast to what happens for stars, the 
solution in the most troublesome part of the numerical domain (i.e., near $x \sim 0, z \sim 0$ ) is not characterized by particularly large values of the fields or of the fluid variables. In support of this conjecture is the evidence that at earlier times, when the shock is closer to the axis, both the absolute errors and the difference between the two formulations are larger.

\section{Conclusion}

A number of astrophysical scenarios can be very conveniently studied numerically by assuming that they possess and preserve a rotation symmetry around an axis. Such an assumption reduces the number of spatial dimensions to be considered and thus the computational costs. This, in turn, allows for a more sophisticated treatment of the physical and astrophysical processes taking place and, as a result, for more realistic simulations.

We have presented a new numerical code developed to solve, in Cartesian coordinates, the full set of general relativistic hydrodynamics equations in a dynamical spacetime and in axisymmetry. More specifically, the new code solves the Einstein equations by using the cartoon method, while HRSC schemes are used to solve the hydrodynamic equations written in a conservative form. An important feature of the code is the use of a novel formulation of the equations of relativistic hydrodynamics in cylindrical coordinates. More specifically, by exploiting a suitable definition of the conserved variables, we removed from the source of the flux-conservative equations those terms, which presented coordinate singularities at the axis, and which are usually retained in the standard formulation of the equations. Despite their simplicity, the changes made to the standard formulation can produce significant improvements on the overall accuracy of the simulations with a truncation error which is often several orders of magnitude smaller.

In order to assess the validity of the new formulation and compare its accuracy with that of the formulation which is most commonly used in Cartesian coordinates, we have performed several tests involving the evolution of oscillating spherical and rotating stars, as well as shock-tube tests. In all cases considered, we have shown that the codes implementing the two formulations yield the expected convergence rate but also that the new formulation is always more accurate, often considerably more accurate, than the standard one.

In view of its simplicity, the new formulation of the equations can be implemented straightforwardly in codes written using the standard formulation and we recommend its use for all situations in which an axisymmetric problem needs to be investigated in full general relativity and in Cartesian coordinates.

\section{Acknowledgments}

It is a pleasure to thank Shin'ichirou Yoshida for providing us with the perturbative eigenfrequencies and Pedro Montero, Olindo Zanotti and Toni Font for useful discussions. The computations were performed on the clusters Peyote, Belladonna and Damiana of the AEI. This work was supported in part by the DFG grant SFB/Transregio 7 and by the JSPS Postdoctoral Fellowship For Foreign Researchers, Grant-in-Aid for Scientific Research (1907803).

\section{Appendix}

In what follows, we recall the essential features of the cartoon method for the solution of the field equations in Cartesian coordinates. Consider therefore the computational domain to have 
extents $0 \leqslant x, z \leqslant d_{\max }$ and $-\Delta y \leqslant y \leqslant \Delta y$, where $d_{\max }$ refers to the location of the outer boundary. Reflection symmetry with respect to the $z=0$ plane can additionally be assumed. The Einstein equations are then solved only on the $y=0$ plane with the derivatives in the $y$-direction being computed with second-order centred stencils using the points at $-\Delta y, 0, \Delta y$.

Taking into account axisymmetry, the rotation in the $(x, y)$ plane is defined as

$$
R(\phi)_{j}^{i}=\left(\begin{array}{ccc}
\cos (\phi) & -\sin (\phi) & 0 \\
\sin (\phi) & \cos (\phi) & 0 \\
0 & 0 & 1
\end{array}\right)
$$

where $R(\phi)^{-1}=R(-\phi)$, and the rotation angle is defined as $\phi=\tan ^{-1}( \pm \Delta y / x)$.

As commented in the main text, the values of all the quantities on the $\pm \Delta y$ planes are computed via rotations of the corresponding values on the $y=0$ planes. More specifically, the components of an arbitrary vector field $T_{i}$ on the $\pm \Delta y$ planes are computed via a $\phi$-rotation as

$$
\begin{aligned}
& T_{x}=T_{x}^{(0)} \cos (\phi)-T_{y}^{(0)} \sin (\phi), \\
& T_{y}=T_{x}^{(0)} \sin (\phi)+T_{y}^{(0)} \cos (\phi), \\
& T_{z}=T_{z}^{(0)},
\end{aligned}
$$

where $T_{i}^{(0)}$ denote the corresponding components at $\left(\sqrt{x^{2}+(\Delta y)^{2}}, 0, z\right)$, which are computed via a Lagrangian interpolation from the neighbouring points on the $x$-axis. Similarly, the components of an arbitrary tensor field $T_{i j}$ tensor will be computed as

$$
\begin{aligned}
& T_{x x}=T_{x x}^{(0)} \cos ^{2}(\phi)-T_{x y}^{(0)} \sin (2 \phi)+T_{y y}^{(0)} \sin ^{2}(\phi), \\
& T_{x y}=\frac{1}{2} T_{x x}^{(0)} \sin (2 \phi)-T_{x y}^{(0)} \cos (2 \phi)+\frac{1}{2} T_{y y}^{(0)} \sin (2 \phi), \\
& T_{y y}=T_{x x}^{(0)} \sin ^{2}(\phi)-T_{x y}^{(0)} \sin (2 \phi)+T_{y y}^{(0)} \cos ^{2}(\phi), \\
& T_{x z}=T_{x z}^{(0)} \cos (\phi)-T_{y z}^{(0)} \sin (\phi), \\
& T_{y z}=T_{x z}^{(0)} \sin (\phi)+T_{y z}^{(0)} \cos (\phi), \\
& T_{z z}=T_{z z}^{(0)}
\end{aligned}
$$

\section{References}

[1] Alcubierre M, Brandt S R, Brügmann B, Holz D, Seidel E, Takahashi R and Thornburg J 2001 Int. J. Mod. Phys. D $10273-89$

[2] Shibata M 2000 Prog. Theor. Phys. $104325-58$

[3] Shibata M 2003 Phys. Rev. D 67024033

[4] Duez M D, Liu Y T, Shapiro S L and Stephens B C 2004 Phys. Rev. D 69104030

[5] Montero P J 2004 Accretion tori arround black holes PhD Thesis SISSA, International School for Advanced Studies

[6] Duez M D, Liu Y T, Shapiro S L and Stephens B C 2005 Phys. Rev. D 72024029

[7] Shibata M and Sekiguchi Y 2005 Phys. Rev. D 72044014

[8] Montero P J, Font J A and Shibata M 2008 arXiv:0805.3099v2 (Phys. Rev. D at press)

[9] Pollney D et al 2007 Phys. Rev. D 76124002

[10] Baiotti L, Hawke I, MonteroP and Rezzolla L 2003 Computational Astrophysics in Italy: Methods and Tools vol 1 ed R Capuzzo-Dolcetta (Trieste: Mem. Soc. Astron. It. Suppl.) p 210

[11] Baiotti L, Hawke I, Montero P J, Löffler F, Rezzolla L, Stergioulas N, Font J A and Seidel E 2005 Phys. Rev. D 71024035 
[12] Baiotti L, Giacomazzo B and Rezzolla L 2008 arXiv: 0804.0594

[13] Goodale T, Allen G, Lanfermann G, Massó J, Radke T, Seidel E and Shalf J 2003 Vector and Parallel Processing-VECPAR'2002, 5th Int. Conf., (Lecture Notes in Computer Science) (Berlin: Springer)

[14] Nakamura T, Oohara K and Kojima Y 1987 Prog. Theor. Phys. Suppl. 90 1-218

[15] Shibata M and Nakamura T 1995 Phys. Rev. D 525428

[16] Baumgarte T W and Shapiro S L 1998 Phys. Rev. D 59024007

[17] York J W 1979 Sources of Gravitational Radiation ed L L Smarr (Cambridge: Cambridge University Press) pp 83-126

[18] Misner C W, Thorne K S and Wheeler J A 1973 Gravitation (San Francisco, CA: Freeman)

[19] Bona C, Massó J, Seidel E and Stela J 1995 Phys. Rev. Lett. 75 600-3

[20] Alcubierre M, Brügmann B, Diener P, Koppitz M, Pollney D, Seidel E and Takahashi R 2003 Phys. Rev. D 67084023

[21] Löffler F, Rezzolla L and Ansorg M 2006 Phys. Rev. D 74104018

[22] Rinne O and Stewart J M 2005 Class. Quantum Grav. 22 1143-66

[23] Ruiz M, Alcubierre M and Núñez D 2008 Gen. Rel. Grav. 40 159-82

[24] Pretorius F 2005 Class. Quantum Grav. 22 425-52

[25] Martí J M, Ibáñez J M and Miralles J A 1991 Phys. Rev. D 433794

[26] Banyuls F, Font J A, Ibáñez J M, Martí J M and Miralles J A 1997 Astrophys. J. 476221

[27] Leveque R J 1992 Numerical Methods for Conservation Laws (Basel: Birkhauser)

[28] Font J A 2003 Living Rev. Rel. 64 (http://www.livingreviews.org/Articles/lrr-2003-4)

[29] Toro E F 1999 Riemann Solvers and Numerical Methods for Fluid Dynamics (Berlin: Springer)

[30] Teukolsky S A 2000 Phys. Rev. D 61087501

[31] Leiler G and Rezzolla L 2006 Phys. Rev. D 73044001

[32] Harten A, Engquist B, Osher S and Chakrabarty S R 1987 J. Comput. Phys. 712311

[33] Colella P and Woodward P R 1984 J. Comput. Phys. 54174

[34] Harten A, Lax P D and van Leer B 1983 SIAM Rev. 2535

[35] Roe P L 1981 J. Comput. Phy. 43357

[36] Aloy M A, Ibáñez J M, Martí J M and Müller E 1999 Astrophys. J. Supp. 122151

[37] Shibata M and Taniguchi K 2006 Phys. Rev. D 73064027

[38] Anderson M, Hirschmann E W, Lehner L, Liebling S L, Motl P M, Neilsen D, Palenzuela C and Tohline J E 2008 Phys. Rev. D 77024006

[39] Liu Y T, Shapiro S L, Etienne Z B and Taniguchi K 2008 Phys. Rev. D 78024012

[40] Oechslin R and Janka H T 2006 MNRAS 3681489

[41] Dumbser M, Enaux C and Toro E F 2008 J. Comput. Phys. 227 3971-4001

[42] Shibata M and Sekiguchi Y I 2003 Phys. Rev. D 68104020

[43] Shibata M, Sekiguchi Y and Takahashi R 2007 Progress of Theoretical Physics 118 257-302

[44] Stergioulas N and Friedman J L 1995 Astrophys. J. 444306

[45] Frauendiener J 2002 Phys. Rev. D 66104027

[46] Font J A, Stergioulas N and Kokkotas K D 2000 Mon. Not. R. Astron. Soc. 313678

[47] Font J A, Goodale T, Iyer S, Miller M, Rezzolla L, Seidel E, Stergioulas N, Suen W M and Tobias M 2002 Phys. Rev. D 65084024

[48] Yo H J, Baumgarte T W and Shapiro S L 2002 Phys. Rev. D 66084026

[49] Dimmelmeier H, Stergioulas N and Font J A 2006 Mon. Not. R. Astron. Soc. 368 1609-30

[50] Yoshida S and Eriguchi Y 2001 Mon. Not. R. Astron. Soc. 322389

[51] Martí J M, Müller E, Font J A, Ibáñez J M and Marquina A 1997 Astrophys. J. 479151 